\title{
Optimal Channel Exploration for Cognitive Radio Networks
}

\author{
Linbo Zhai ${ }^{1, a}$ and Feng Yang ${ }^{1, b}$ \\ ${ }^{1}$ Shandong Provincial Key Laboratory for Distributed Computer Software \\ Novel Technology, \\ Shandong Normal University, \\ Jinan, China, 250014

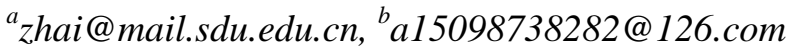

\begin{abstract}
In this paper, the optimal channel exploration is proposed for cognitive radio networks. In the networks, primary users (PUs) utilize the channels with fixed slot structure. Cognitive users (CUs) should sense the channels within each slot. We study the relationship of the channel states between the nearby slots and range channels by the decreasing idle probabilities of all channels. Then we use stop rule to address the channel exploration problem, which makes CUs to access the optimal idle channel. The simulation results show our proposed scheme can obtain higher throughput.

Keywords: Cognitive radio; Channel exploration; Stop rule; Network; Throughput

\section{Introduction}

Cognitive radio is the new technology to improve the channel utilization. In this cognitive system, primary users (PUs) use the channel with one or several whole slots. Therefore, cognitive users (CUs) should sense channels to find the idle ones. Because of the hard limit, the CUs can only sense one channel at a time. There have been channel exploring works in [1, 2], which assume the channel state distributions are fixed. However, the channel states (idle or busy) are related
\end{abstract}


between nearby slots in fact [3, 4]. Considering the channel state relationship and the order in which CUs sense the channels and the channel exploring problem is researched in [5], where the energy consumption is ignored in the sensing process. In [6, 7], energy consumption is considered but channel state relationship is neglected.

In this paper, the optimal channel exploration is proposed for cognitive radio networks. We study the relationship of the channel states between the nearby slots and range channels by the decreasing idle probabilities of channels. Then we use stop rule to address the channel exploration problem, which makes CUs to access the optimal idle channel to maximize the throughput. The simulation results show our proposed scheme can obtain higher throughput.

\section{Channel States}

In the cognitive network, channel states changes following the slot structure. From a long time observation, we can derive the channel state relationship between nearby slots for each slot. There are four state transition probabilities (idle to idle, idle to busy, busy to idle, busy to busy) for any channel across two adjoining slot. Let 0 denote an idle state, and 1 denote a busy state. For channel $i$, the state transition matrix $P_{i}$ is

$$
P_{i}=\left[\begin{array}{ll}
p_{i 00} & p_{i 01} \\
p_{i 10} & p_{i 11}
\end{array}\right]
$$

For each channel, there are initial probabilities about its channel states (busy or idle). Let $u_{i, 0}$ denote the probability that the channel $i$ is busy (used by PUs), and $u_{i, 1}$ denote the probability that the channel $i$ is idle. According to the initial probabilities and the state transition matrix, we can compute the channel state probability of channel $i$ in the current slot as follows.

$$
Q_{i}=\left[\begin{array}{ll}
Q_{i, 0} & Q_{i, 1}
\end{array}\right]=\left[\begin{array}{ll}
u_{i, 0} & u_{i, 1}
\end{array}\right] P_{i}^{n}
$$


where $n$ denotes the step number from the initial state to the current state, $Q_{i}$ denotes the channel state probability vector of channel $i, Q_{i, 0}$ denotes the idle probability of channel $i$ and $Q_{i, 0}$ denotes the idle probability of channel $i$ in the current slot.

For each slot, we can compute the channel state probability vectors of the whole channels. Then we range all channels with the decreasing idle probability order. When CUs begin to sense the channels, they should follow the decreasing idle probability order. Therefore, CUs could find an idle channel to access as soon as possible.

In addition, the state transition matrix $P_{i}$ for each channel $i$ is not fixed. According to each sensing, the state transition matrix can be renewed with the sensing outcomes.

\section{Channel Exploring}

When a slot begins, the idle probabilities of the whole channels can be computed. Then we range all channels with the decreasing idle order. CUs sense the channels following the decreasing idle order. As a $\mathrm{CU}$, it wants to find an idle channel to maximize the throughput. In this paper, we use stop rule to realize this purpose. For each channel, there is the signal-noise-ratio (SNR) which can be assumed following independent identically distribution. Let $T$ denote the duration of each slot, $\delta$ denote the sensing time for each channel, $P O_{1}$ denote the consumed power for one channel sensing and $\mathrm{PO}_{2}$ denote the consumed power for the data transmission. When a CU senses $n$ channels, if there are not less than one idle channel, it will find an idle channel to maximize its throughput. The throughput is

$$
y_{n}\left(S N R_{\max }\right)=\frac{(T-n \delta) \log \left(1+S N R_{\max }\right)}{n \delta P O_{1}+(T-n \delta) P O_{2}}
$$

where $S N R_{\max }$ denotes the maximum of SNRs among the idle channels.

Then the CU computes the expected throughput $E\left[Y_{n+1} \mid y_{n}\right]$ after sensing the next channel $n+1$. From (2), the channel state probability of channel $n+1$ can be 
computed in the current slot as $Q_{n+1}=\left[Q_{n+1,0} Q_{n+1,1}\right]$, where $Q_{n+1,0}$ denotes the channel $n+1$ is idle and $Q_{n+1,1}$ denotes the channel $n+1$ is busy. There may be three cases:

i) if the channel $n+1$ is busy (used by PUs), the expected throughput $E\left[Y_{n^{+}}\right.$ $\left.{ }_{1} \mid y_{n}\right]=y_{n}\left(S N R_{\max }\right)$;

ii) if the channel $n+1$ is idle and the $S N R_{n+1}$ is less than $S N R_{\max }$, the expected throughput $E\left[Y_{n+1} \mid y_{n}\right]=y_{n+1}\left(S N R_{\max }\right)$;

iii) if the channel $n+1$ is idle and the $S N R_{n+1}$ is no less than $S N R_{\max }$, the expected throughput $E\left[Y_{n+1} \mid y_{n}\right]=y_{n+1}\left(S N R_{n+1}\right)$;

Therefore, we can obtain the expected throughput $E\left[Y_{n+1} \mid y_{n}\right]$ as

$$
\begin{aligned}
E\left[Y_{n+1} \mid y_{n}\right]= & Q_{i+1,0} \frac{(T-n \delta-\delta) \log \left[1+\max \left(S N R_{\max }, S N R_{n+1}\right)\right]}{(n+1) \delta P O_{1}+(T-n \delta-\delta) P O_{2}} \\
& +Q_{i+1,1} \frac{(T-n \delta-\delta) \log \left[1+S N R_{\max }\right]}{(n+1) \delta P O_{1}+(T-n \delta-\delta) P O_{2}}
\end{aligned}
$$

When a CU senses $n$ channels and obtains the throughput $y_{n}\left(S N R_{\max }\right)$, we can compute the expected throughput $E\left[Y_{n^{+}} \mid y_{n}\right]$ after sensing the next channel $n+1$ by (4).

This paper uses one -stage looking stop to find the optimal idle channel. If the expected throughput $E\left[Y_{n+1} \mid y_{n}\right]$ is less than $y_{n}\left(S N R_{\max }\right)$, the CU should stop and the channel $n$ is the proper channel to access. Otherwise, if the expected throughput $E\left[Y_{n+1} \mid y_{n}\right]$ is bigger than $y_{n}\left(S N R_{\max }\right)$, the CU should sense the channel $n+1$ to find the optimal channel until the last channel.

\section{Simulation Results and Discussions}


In this section, the parameters are set as follows: the duration of a slot is $0.1 \mathrm{~s}$ ( $T=0.1 \mathrm{~s})$, the power of data transmission is $1 \mathrm{w}\left(\mathrm{PO}_{2}=1 \mathrm{w}\right)$ and the sensing time of each channel is $0.005 \mathrm{~s}$ ( $\delta=0.005 \mathrm{~s}$ ).

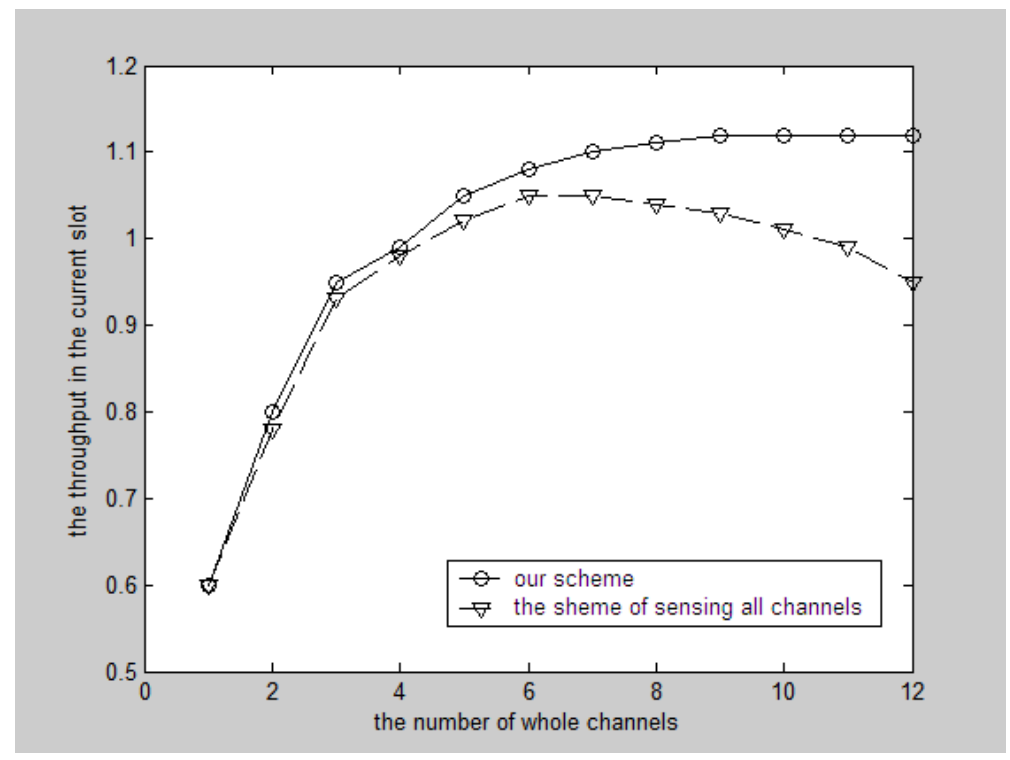

Fig. 1 the throughput of our scheme and sensing all channels with $P O_{1}=0.3 \mathrm{w}$

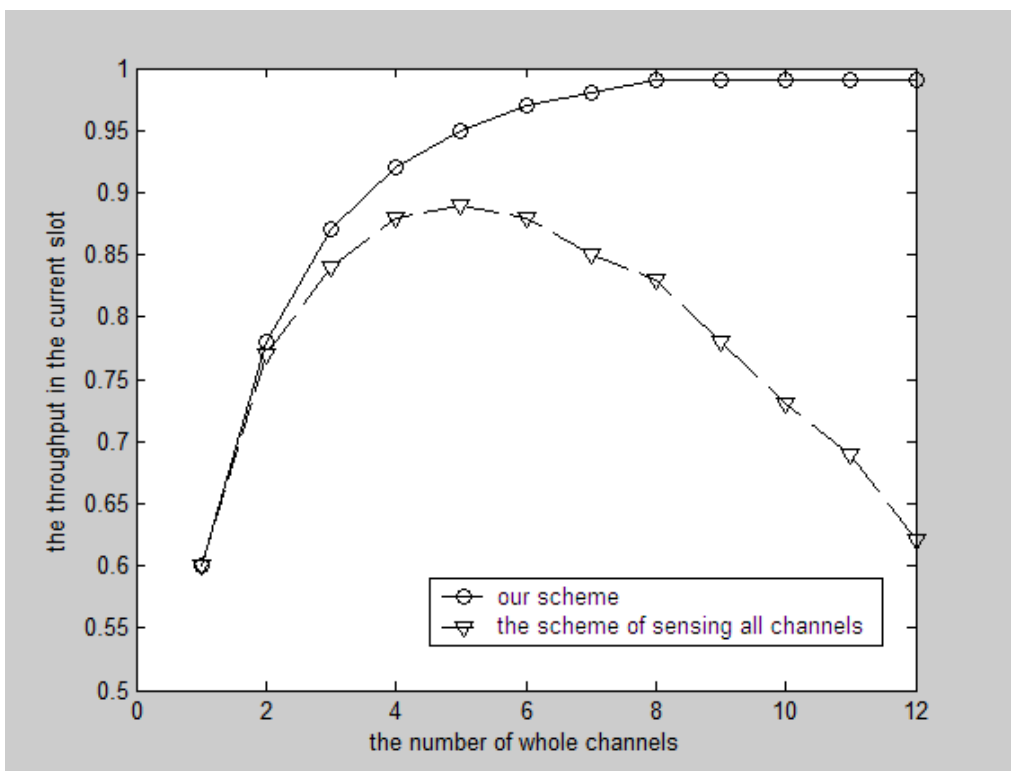

Fig. 2 the throughput of our scheme and sensing all channels with $P O_{1}=0.8 \mathrm{w}$ 
We compare the throughput of our scheme using one-step looking stop with that of the scheme of sensing all channels. Here, it is assumed all channels are idle. In Fig. 1, the consumed power of one channel sensing is $0.3 \mathrm{w}\left(P O_{1}=0.3 \mathrm{w}\right)$, and in Fig. 2, the consumed power of one channel sensing is $0.8 \mathrm{w}\left(\mathrm{PO}_{1}=0.8 \mathrm{w}\right)$. From Fig 1 and Fig. 2, we can see our scheme can obtain higher throughput than the scheme of sensing all channels in the current slot. The reason is that our scheme makes CUs sensing less channels and the sensing time is saved for data transmission. Compared Fig. 1 with Fig. 2, the throughput is decreased when the sensing power increases.

\section{Conclusion}

This paper proposes the optimal channel exploration for cognitive radio networks. We study the relationship of the channel states between the nearby slots and range channels by the decreasing idle probabilities. Then we use one-step looking stop rule to address the channel exploration problem, which makes CUs to access the optimal idle channel. The simulation results show our proposed scheme can obtain higher throughput.

\section{References}

[1] H. Jiang, L. Lai, R. Fan, and H. V. Poor, “Optimal selection of channel sensing order in cognitive radio,” IEEE Trans. Wireless Commun., 8(1), pp. 297-307, 2009.

[2] H. T. Cheng and W. Zhuang, "Simple channel sensing order in cognitive radio networks,” IEEE J. Sel. Areas Commun., 29(4), pp. 1-13, 2011.

[3] B. J. Kang, "Spectrum sensing issues in cognitive radio networks," in Proc. 2009 Intl. Sym. Comm. Info. Tech., pp. 824-828, 2009. 
[4] M. Lopez-Benitez and F. Casadevall, "Modeling and simulation of time correlation properties of spectrum use in cognitive radio," in Proc. 2011 Intl. Conf. Cog. Radio Oriented Wireless Net. and Commun., 2011.

[5] U. G and A. P. Kannu, "Throughput optimal multi-slot sensing procedure for a cognitive radio”, IEEE Comm. Lett., 17(12), pp. 2292-2295, 2013.

[6] Y. Pei, Y.-C. Liang, K. C. Tech, and K. H. Li, "Energy-efficient design of sequential channel sensing in cognitive radio networks: optimal sensing strategy, power allocation and sensing order”, IEEE J. Sel. Areas Commun., 29(8), pp. 1648-1659, 2011.

[7] Y. Xu, J. Wang, Z. Zhang, A. Anpalagan and L. Shen, "Optimal energy-efficient channel exploration for opportunistic spectrum usage”, IEEE Wireless Commu. Let., 1(2), pp. 77-80, 2012. 